\title{
Sintomas depressivos em adolescentes com excesso de peso
}

\author{
Depressive symptoms in overweight adolescentes \\ Síntomas depresivos en adolescentes con sobrepeso
}

Recebido: 13/07/2021 | Revisado: 21/07/2021 | Aceito: 23/07/2021 | Publicado: 31/07/2021

\author{
Rafaela Gois de Mendonça \\ ORCID: https://orcid.org/0000-0002-8982-5564 \\ Universidade Federal de Sergipe, Brasil \\ E-mail: rafaelagoisenf@gmail.com \\ Glebson Moura Silva \\ ORCID: https://orcid.org/0000-0002-4977-2787 \\ Universidade Federal de Sergipe, Brasil \\ E-mail: glebsonmoura@yahoo.com.br \\ Bruno Ferreira Amorim \\ ORCID: https://orcid.org/0000-0002-2581-2983 \\ Universidade Federal de Sergipe, Brasil \\ E-mail: brunofamorim@hotmail.com.br \\ Marina de Souza Lima \\ ORCID: https://orcid.org/0000-0002-0100-5714 \\ Universidade Federal de Sergipe, Brasil \\ E-mail: marii2406.ml@gmail.com \\ Danielly Cardoso de Mesquita \\ ORCID: https://orcid.org/0000-0002-5551-1479 \\ Universidade Federal de Sergipe, Brasil \\ E-mail: danielly_2802@hotmail.com \\ Caroline Stefany Menezes Ferreira Araújo \\ ORCID: https://orcid.org/0000-0002-5129-3478 \\ Universidade Federal de Sergipe, Brasil \\ E-mail: caroline.ferreira.1802@gmail.com \\ Anne Manuelle dos Santos \\ ORCID: https://orcid.org/0000-0002-2049-8593 \\ Universidade Federal de Sergipe, Brasil \\ E-mail: annemanuelle63@gmail.com \\ Andreia Freire de Menezes \\ ORCID: https://orcid.org/0000-0002-2972-8236 \\ Universidade Federal de Sergipe, Brasil \\ E-mail: deiamenezes1@hotmail.com
}

\section{Resumo}

Objetivo: analisar a influência que sintomas depressivos tem sobre e a obesidade ou excesso de peso, delimitando se aspectos como gênero e fatores sociais apresentam relação direta com o quadro. Metodologia: foram realizadas pesquisas na Biblioteca Virtual da Saúde a qual engloba as seguintes bases de dados: Base de Dados de Enfermagem (BDENF), Sistema de Análise e Recuperação da Literatura Médica (MEDLINE) e a Literatura Latino-Americana e do Caribe em Ciências da Saúde (LILACS). Resultado: a amostra final consistiu em 13 artigos, sendo o mais antigo publicado em 2015 e o mais recente em 2020. Conclusão: o presente estudo auxilia na compreensão dos principais fatores apontados na literatura como percursores da relação entre sintomas depressivos e obesidade.

Palavras-chave: Sobrepeso; Obesidade; Depressão; Adolescente.

\begin{abstract}
Objective: to analyze the influence that depressive symptoms have on obesity or excess weight, delimiting whether aspects such as gender and social factors are directly related to the condition. Methodology: research was carried out in the Virtual Health Library which includes the following databases: Nursing Database (BDENF), Analysis and Recovery System of Medical Literature (MEDLINE) and Latin American and Caribbean Literature in Sciences of Health (LILACS). Result: the final sample consisted of 13 articles, the oldest being published in 2015 and the most recent in 2020. Conclusion: the present study helps to understand the main factors identified in the literature as precursors of the relationship between depressive symptoms and obesity.
\end{abstract}

Keywords: Overweight; Obesity; Depression; Adolescent. 


\section{Resumen}

Objetivo: analizar la influencia que los síntomas depresivos tienen sobre la obesidad o el sobrepeso, delimitando si aspectos como el género y los factores sociales están directamente relacionados con la condición. Metodología: la investigación se realizó en la Biblioteca Virtual en Salud que incluye las siguientes bases de datos: Base de Datos de Enfermería (BDENF), Sistema de Análisis y Recuperación de Literatura Médica (MEDLINE) y Literatura Latinoamericana y Caribeña en Ciencias de la Salud (LILACS). Resultado: la muestra final estuvo conformada por 13 artículos, siendo el más antiguo publicado en 2015 y el más reciente en 2020. Conclusión: el presente estudio ayuda a comprender los principales factores identificados en la literatura como precursores de la relación entre síntomas depresivos y obesidad.

Palabras clave: Sobrepeso; Obesidad; Depresión; Adolescente.

\section{Introdução}

A infância e a adolescência são estágios que apresentam um papel fundamental no desenvolvimento dos aspectos que levam a moldar a vida adulta de cada indivíduo (Salles, 2005). Em uma perspectiva global, há dois fatores preocupantes que atuam na saúde dos adolescentes: a obesidade e a depressão.

A obesidade é uma patologia crônica ocasionada pela reserva em demasia de gordura no organismo. Segundo a Organização Mundial da Saúde (OMS), os casos sobre a obesidade vêm aumentando nos últimos 40 anos, principalmente em países em desenvolvimento. No Brasil, esse crescente revela uma taxa de $14 \%$ crianças e adolescentes obesos, na faixa etária entre os 5 e 19 anos (OMS, 2019).

A depressão está dentro de um grupo de doenças psiquiátricas caracterizado como transtornos de humor. Esse tipo de alteração psíquica envolve mudanças fisiológicas que comprometem as relações interpessoais, práticas diárias e as funções vegetativas do indivíduo (Sadock, 2016). Os mais diversos aspectos influem para a predisposição de sintomas depressivos, tais como sexo, idade e fatores socioeconômicos.

Em relação a depressão o panorama mundial é em torno de 300 milhões de pessoas afetadas. No Brasil, 5,8\% da população apresenta sintomas depressivos (OMS, 2017). Segundo a Organização das Nações Unidas (ONU), a maior parte desses transtornos mentais, como os de humor, começam aos 14 anos (ONU, 2019).

Evidências científicas apontam que crianças e adolescentes obesos apresentam uma alta prevalência de sintomas depressivos, com uma metanálise realizada na china encontrando prevalência da ordem de 24,02\% em sua população com essas características (Rao, 2019). Durante a adolescência há uma construção da autopercepção de maneira que a satisfação com a imagem corporal pode influenciar comportamento social e na dinâmica alimentar do indivíduo, com muitos transtornos alimentares surgindo neste período assim como transtornos psicológicos advindos dessa autopercepção.

Além disso, fatores como compulsão alimentar por um mecanismo compensatório e menor autocuidados com o corpo gerados pela depressão podem levar a um aumento do peso em um ciclo de retroalimentação positiva entre obesidade e depressão, ou seja, pacientes que apresentam desordem de humor utilizam a ingestão de alimentos em excesso como um mecanismo de enfrentamento (Goldschmidt, 2015). Nos adolescentes, a percepção que se tem do peso aparente pode ser tornar um fator a ser analisado para possível desenvolvimento de sintomas depressivos, pois é nessa fase onde a cultura social do perfeccionismo corporal se faz mais presente. Dessa maneira, aqueles adolescentes que não se enquadram nas perspectivas sociais apresentam uma maior probabilidade de desenvolverem sintomas depressivos (Quek, 2017).

A relevância deste estudo está sustentada na busca pela associação entre obesidade e depressão, pois devido à alta prevalência de transtornos de humor em jovens e o impacto dessa doença em suas vidas, a identificação da obesidade como um fator de risco importante e a determinação das relações bidirecionais dessas doenças entre si podem proporcionar uma melhor compreensão e, por conseguinte, melhores estratégias de intervenção e tratamento dessas condições quando associadas (Lindberg, 2020). 
Research, Society and Development, v. 10, n. 9, e48910918185, 2021

(CC BY 4.0) | ISSN 2525-3409 | DOI: http://dx.doi.org/10.33448/rsd-v10i9.18185

Essa revisão integrativa teve como objetivo analisar a influência que sintomas depressivos tem sobre e a obesidade ou excesso de peso, delimitando se aspectos como gênero e fatores sociais apresentam relação direta com o quadro.

\section{Metodologia}

Essa pesquisa aborda uma revisão integrativa, a qual é definida por meio do amplo nicho metodológico que inclui pesquisas experimentais e não-experimentais (Soares, 2014). Dessa maneira, foi realizado um levantamento de dados organizado em etapas que sucederam às escolhas dos artigos em análise.

A construção do estudo baseou-se em seis etapas: 1. Elaboração da pergunta norteadora da pesquisa; 2. Busca na base de dados através dos descritores definidos; 3 . Triagem para seleção dos artigos em acordo com a pergunta norteadora; 4. Leitura crítica dos estudos selecionados; 5. Análise dos resultados obtidos e 6. Súmula das informações coletadas.

A primeira etapa caracteriza-se pela pergunta norteadora. Esta, por sua vez, foi construída através dos principais pontos a serem abordados sobre o assunto: quais os fatores associados na relação entre sintomas depressivos e obesidade em adolescentes?

Em seguida, na segunda etapa, foram realizadas pesquisas na Biblioteca Virtual da Saúde a qual engloba as seguintes bases de dados: Base de Dados de Enfermagem (BDENF), Sistema de Análise e Recuperação da Literatura Médica (MEDLINE) e a Literatura Latino-Americana e do Caribe em Ciências da Saúde (LILACS). Os descritores utilizados foram relativos à temática, seguindo as seguintes combinações: obesidade (sobrepeso or obesidade) AND depressão (depressão) AND adolescentes (adolescentes or adolescência). A seleção dos descritores foi norteada por sua proximidade ao objeto em questão.

Ademais, a busca foi norteada através dos seguintes critérios de inclusão: estudos que relacionaram sintomas depressivos à obesidade e sobrepeso em adolescentes inseridos na faixa etária estabelecida pela OMS que é de 10 aos 19 anos. Foram, também, considerados estudos publicados em outros idiomas e em diferentes anos. Além disso, foram excluídos os estudos que não se encontravam disponíveis na íntegra nas fontes pesquisadas e que apresentavam uma faixa etária para adolescentes diferente da estabelecida pela organização. A busca foi realizada em novembro de 2020.

Na terceira etapa, que correspondeu ao início da triagem, foram obtidos como resultados iniciais, através dos descritores, 1067 artigos, dos quais ao serem analisados por títulos foram selecionados 189. Na quarta etapa, posteriormente, esses foram analisados e filtrados de acordo com as informações contidas nos resumos. Assim, aqueles que continham maior relevância para o estudo foram selecionados, obtendo-se 53 resumos que estavam associados ao tema da pesquisa e se enquadram nos critérios de inclusão.

A quinta etapa, baseou-se na leitura completa e na análise crítica, sendo selecionados apenas os estudos que se encontravam de acordo com os critérios de inclusão, ou seja, que possuíam conteúdo relacionado com a temática. Dessa forma, foram excluídos 40 artigos que não se encontravam nos critérios de inclusão do estudo sendo selecionados 13 apenas conforme Figura 1.

A sexta etapa foi composta pela organização e sumarização das principais informações que contou com as seguintes variáveis: número do artigo, título, autores, país de estudo, ano de publicação, tipo de estudo, objetivo do estudo, tamanho da amostra e conclusão do estudo. E a sétima e última etapa foi realizada através da síntese dos estudos para agrupar o conhecimento acerca do problema da pesquisa. 
Figura 1: Fluxograma do Processo de Busca e Seleção dos Artigos.

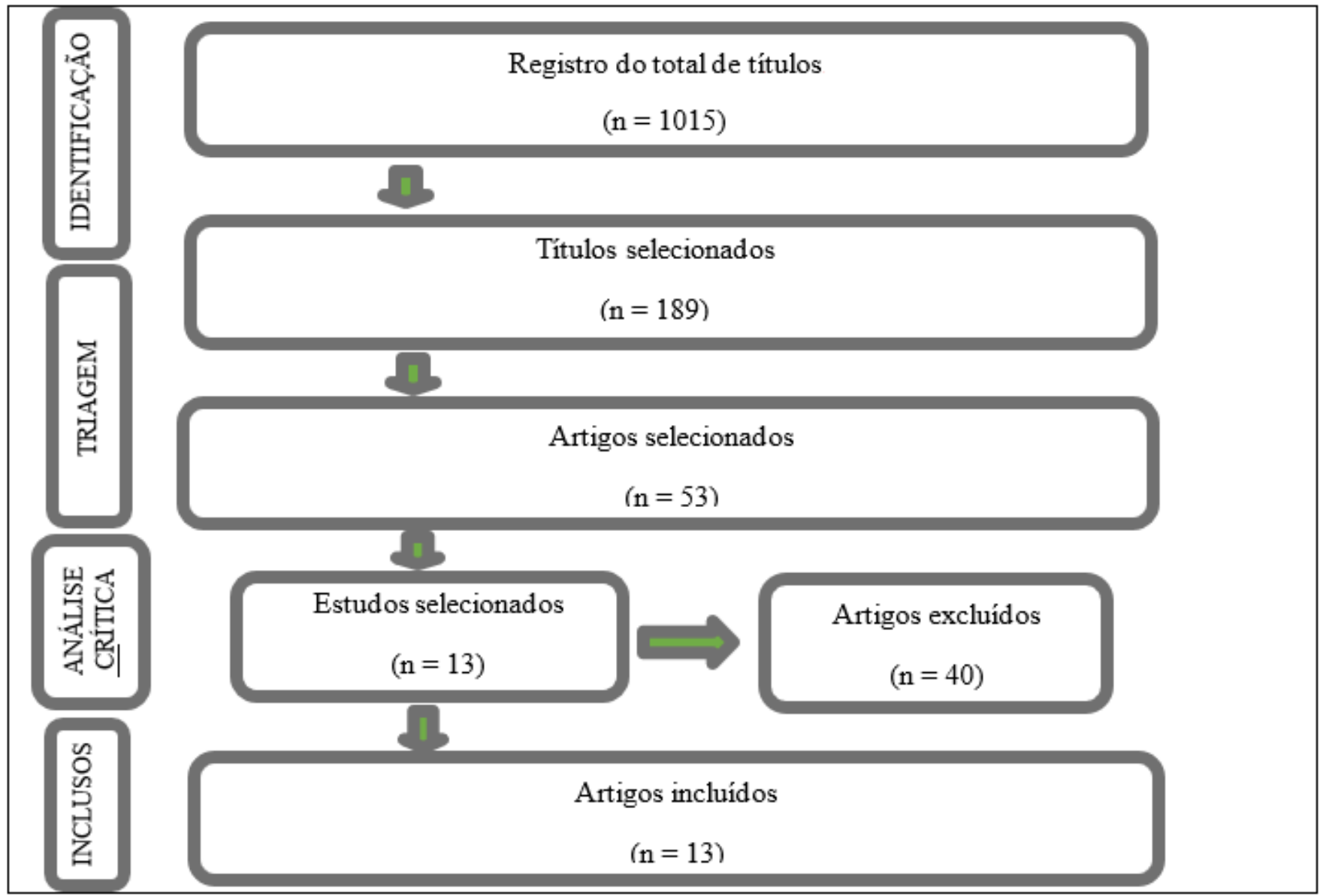

Fonte: Autores.

\section{Resultados}

A amostra final consistiu em 13 artigos, sendo o mais antigo publicado em 2015 e o mais recente em 2020, dos quais, 15,38\% foram publicados 2018; 15,38\% entre 2015; 15,38\% em 2020; 23,07\% em 2019; 30,77\% em 2017.

Em relação ao idioma dos estudos, 69,23\% foram publicados em inglês; 23,08\% em espanhol; e 7,69\% em português. Quanto ao país de realização do estudo, 30,77\% foram realizados nos Estados Unidos da América, 15,38\% na China e 7,69\% no Brasil, assim como no México, Irã, Equador, Suécia e Singapura.

Os delineamentos mais frequentes foram o estudo transversal $(46,15 \%)$, estudo de coorte $(23,07 \%)$, meta-análise $(15,38 \%)$, exploratório $(7,69 \%)$ e longitudinal $(7,69 \%)$. O somatório total da amostra dos estudos consistiu de 28654 participantes.

Quatro artigos demonstraram que a insatisfação corporal gerada pela obesidade/sobrepeso é o principal fator estressor associado à depressão nos pacientes avaliados. Além disso, em um estudo foi encontrado que a obesidade central e não a geral (IMC) era um preditor positivo para sintomas depressivos em adolescentes (Tabela 1). Muitos desses artigos encontraram maior incidência dessa insatisfação corporal em meninas adolescentes e um artigo encontrou um dado interessante sobre insatisfação corporal, na qual a percepção equivocada sobre a própria aparência corporal em crianças obesas atuava como fator protetor para esses indivíduos (Tabela 2). 
Tabela 1: artigos demonstraram a associação entre obesidade/sobrepeso com depressão e insatisfação corporal.

\begin{tabular}{|c|c|c|c|c|}
\hline $\mathbf{N}^{\mathbf{o}}$ & Titulo & Ano & $\beta$ & p valor \\
\hline 1 & $\begin{array}{l}\text { Central or overall obesity: Which one is a better predictor of depressive } \\
\text { symptoms in children, adolescents, and youths? }\end{array}$ & 2018 & $0,13 * *$ & $\mathrm{p}<0,05$ \\
\hline 2 & $\begin{array}{l}\text { Mediators involved in the relation between depressive symptoms and } \\
\text { weight status in female adolescents and young adults }\end{array}$ & 2015 & 0,038 & 0,007 \\
\hline 3 & $\begin{array}{l}\text { Longitudinal bidirectional relations between body dissatisfaction and } \\
\text { depressive symptoms among Black adolescents: A cross-lagged panel } \\
\text { analysis }\end{array}$ & 2020 & $\begin{array}{l}\mathrm{T} 1-\mathrm{T} 2: \beta= \\
0,42 \\
\mathrm{~T} 2-\mathrm{T} 3: \beta= \\
0,36\end{array}$ & $\mathrm{p}<0,001$ \\
\hline 4 & $\begin{array}{l}\text { Autoestima, imagem corporal e depressão de adolescentes em diferentes } \\
\text { estados nutricionais }\end{array}$ & 2017 & $\begin{array}{c}\text { Diferença } \\
-2,5 ; \pm 1.3\end{array}$ & $\begin{array}{c}\mathrm{p} \\
<0,0001\end{array}$ \\
\hline
\end{tabular}

$\boldsymbol{\beta}-\beta$ padronizado. T1-T2-T3: indicam respectivamente passagens temporais entre as avaliações

** - esse estudo encontrou a obesidade central como preditor de sintomas depressivos em adolescentes e não a obesidade geral. Fonte: Autores.

Tabela 2: estudos com análises diferentes de risco relativo para depressão em obesos e falsa percepção corporal como fator protetor da depressão em obesos.

\begin{tabular}{|c|c|c|c|c|}
\hline $\mathbf{N}^{\mathbf{o}}$ & Titulo & Ano & $R R$ & p valor \\
\hline 5 & $\begin{array}{l}\text { Asociación entre índice de masa corporal y depresión en mujeres } \\
\text { adolescentes }\end{array}$ & 2017 & 8,55 & $\mathrm{p}<0,05$ \\
\hline \multirow[t]{2}{*}{6} & \multirow{2}{*}{$\begin{array}{l}\text { Cross-sectional and Prospective Examination of Weight Misperception } \\
\text { and Depressive Symptoms Among Youth with Overweight and Obesity }\end{array}$} & \multirow[t]{2}{*}{2017} & $\boldsymbol{\beta}$ & \multirow[t]{2}{*}{$\mathrm{p}<0,05$} \\
\hline & & & $\beta=-4,43$ & \\
\hline
\end{tabular}

$\mathrm{RR}-$ Risco relativo. $\boldsymbol{\beta}-\beta$ padronizado. Fonte: Autores.

Vários artigos encontraram altas taxas de prevalência da depressão em adolescentes obesos/sobrepeso. Além disso, adolescentes obesos apresentaram muito mais chances de apresentarem sintomas depressivos que indivíduos com peso normal, com a razão de chances variando de 1,34 a 17,94 entre os estudos. Em um estudo não foi encontrado resultados estatisticamente significativos para essa correlação (Tabela 3).

Tabela 3: artigos que trouxeram dados sobre prevalência e razões de chances para depressão em obesos.

\begin{tabular}{clcccc}
\hline $\mathbf{N}^{\mathbf{0}}$ & \multicolumn{1}{c}{ Titulo } & Ano & $\mathbf{P}$ & OR & p valor \\
\hline 7 & $\begin{array}{l}\text { Asociación de sobrepeso u obesidad con trastornos del estado de ánimo } \\
\text { en adolescentes }\end{array}$ & 2018 & $83,3 \%$ & 17,94 & $\mathrm{p}=0,000$ \\
\hline 8 & Does major depression affect risk for adolescent obesity? & 2015 & N/A & 2,87 & $\mathrm{p}<0,05$ \\
\hline 9 & $\begin{array}{l}\text { Exploring the association between childhood and adolescent obesity and } \\
\text { depression: a meta-analysis }\end{array}$ & 2017 & N/A & 1,34 & $\mathrm{p}=0,005$ \\
\hline 10 & $\begin{array}{l}\text { Anxiety and depression in children and adolescents with obesity: a } \\
\text { nationwide study in Sweden }\end{array}$ & 2020 & $9,7 \%$ & $\mathrm{~F}=1,43$ & $\mathrm{p}<0,0001$ \\
\hline 11 & Depresión como factor asociado a la obesidad en adolescentes & 2019 & N/A & 4,7 & $\mathrm{p}=0,578^{*}$ \\
\hline 12 & $\begin{array}{l}\text { Prevalence of depressive symptoms in overweight and obese children } \\
\text { and adolescents in mainland China: A meta-analysis of comparative } \\
\text { studies and epidemiological surveys }\end{array}$ & 2019 & $24,02 \%$ & 1,877 & $\mathrm{p}<0,001$ \\
\hline 13 & $\begin{array}{l}\text { Association between depression and overweight in Chinese adolescents: } \\
\text { a cross-sectional study }\end{array}$ & 2019 & $13,15 \%$ & 1.47 & $\mathrm{p}=0,004$ \\
\hline
\end{tabular}

P - Prevalência da depressão em obesos encontrada.

OR - Odds Ratio da depressão em obesos.

F- Feminino. M-Masculino.

* - O dado não foi estatisticamente significativo. N/A - Não se aplica.

Fonte: Autores. 
Quadro 4: Principais conclusões e referências dos estudos. Lagarto, Sergipe, Brasil, 2020.

\begin{tabular}{|c|c|c|}
\hline $\mathrm{N}^{\mathrm{o}}$ & Autores & Principais conclusões \\
\hline 1 & ESMAEILZADEH, $\mathrm{S}$. & $\begin{array}{l}\text { A obesidade central (mas não a obesidade geral) foi um preditor significativo de SD em crianças e adolescentes (7- } \\
18 \text { anos). No entanto, a SD em jovens (19-24 anos) não foi significativamente associada aos índices de obesidade } \\
\text { corporal central e geral. }\end{array}$ \\
\hline 2 & GOLDSCHMIDT, A. B. & $\begin{array}{l}\text { Relata mediadores, como a má qualidade da dieta em adolescentes, que influencia na ligação entre os sintomas } \\
\text { depressivos e a obesidade. O estudo concluiu que a compulsão alimentar é um mecanismo utilizado pelos } \\
\text { adolescentes para compensar os sintomas depressivos. }\end{array}$ \\
\hline 3 & WANG Y. & $\begin{array}{l}\text { As relações recíprocas de sintomas depressivos-insatisfação corporal entre adolescentes negros com sobrepeso / } \\
\text { obesidade, mas não suas contrapartes de peso saudável, sugerem que o sobrepeso / obesidade é um estressor no } \\
\text { desenvolvimento de distúrbios psicológicos graves, por exemplo, insatisfação corporal e sintomas depressivos entre } \\
\text { negros adolescentes iniciais, semelhante a adolescentes de outros grupos raciais / étnicos. }\end{array}$ \\
\hline 4 & $\begin{array}{l}\text { RENTZ-FERNANDES, } \\
\text { A. R. }\end{array}$ & $\begin{array}{l}\text { Nesse artigo, é apresentado dois modelos, que abordam a relação da depressão com autoestima, os quais por meio } \\
\text { dos resultados obtidos induz-se que o mais adequado é relacionar que os sintomas depressivos são mais suscetíveis } \\
\text { quando relacionados a percepções associadas a baixa autoestima. }\end{array}$ \\
\hline 5 & $\begin{array}{l}\text { OCAMPO BUSTOS, J. } \\
\text { E. }\end{array}$ & $\begin{array}{l}\text { O autor expõe a relação entre o excesso de peso e a autoestima dos jovens, bem como retrata a ligação da obesidade } \\
\text { com a depressão ao expressar que essa relação compreende, entre outros fatores, a percepção da imagem corporal e } \\
\text { da auto concepção geral e física do adolescente. }\end{array}$ \\
\hline 6 & THURSTON, I. B. & $\begin{array}{l}\text { Esse estudo revela que os sintomas depressivos e a obesidade estão interligados através da percepção negativa que } \\
\text { o adolescente obeso tem sobre seu peso. Exibe, também, esse ponto de vista por meio de uma estratificação étnica. }\end{array}$ \\
\hline 7 & AGUILAR, C. K.. & $\begin{array}{l}\text { O artigo traz como conclusão a existência de uma relação entre a obesidade e os transtornos de humor, como a } \\
\text { depressão. Entre seus resultados foram apresentados dados que sustentam o aumento na probabilidade de transtornos } \\
\text { de humor estarem diretamente ligados ao excesso de peso e obesidade. }\end{array}$ \\
\hline 8 & $\begin{array}{l}\text { ROBERTS, R. E.; } \\
\text { DUONG, H. T. }\end{array}$ & $\begin{array}{l}\text { A correlação entre a depressão e a obesidade nesse artigo é tratada de maneira indireta e explicada por meio de um } \\
\text { fator determinante que é a imagem corporal. É exposto que o peso percebido pelos adolescentes é mais responsável } \\
\text { pelos sintomas depressivos do que o excesso de gordura. }\end{array}$ \\
\hline 9 & QUEK, Y. H. & $\begin{array}{l}\text { Foi inferido nesse estudo que crianças e adolescentes obesos apresentam maiores probabilidades de desenvolverem } \\
\text { sintomas depressivos e traz como fator a insatisfação corporal, a qual é predisposta por meio de fatores sociais. }\end{array}$ \\
\hline 10 & LINDBERG L. & $\begin{array}{l}\text { Os resultados apoiam a hipótese de que a obesidade per se está associada ao risco de ansiedade e depressão em } \\
\text { crianças e adolescentes. A ansiedade e a depressão causam estresse e sofrimento emocional e fisiológico e também } \\
\text { podem dificultar o tratamento da obesidade. }\end{array}$ \\
\hline 11 & $\begin{array}{l}\text { GUTIÉRREZ- } \\
\text { SÁNCHEZ G. }\end{array}$ & O estudo não obteve um dado estatístico que sugira que obesidade e depressão estão relacionadas. \\
\hline 12 & RAO, WW. & $\begin{array}{l}\text { Este estudo descobriu que os sintomas depressivos são comuns em crianças e adolescentes com sobrepeso e obesos } \\
\text { na China }\end{array}$ \\
\hline 13 & ZHAO, Z. & $\begin{array}{l}\text { O achados fornecem evidências de que a depressão está associada ao sobrepeso ou obesidade entre adolescentes na } \\
\text { China, especialmente entre os homens. }\end{array}$ \\
\hline
\end{tabular}

SD- Sintomas Depressivos.

Fonte: Autores.

A maioria dos estudos incluídos relatou a associação entre obesidade e sintomas depressivos na adolescência principalmente relacionados a presença de baixa autoestima em jovens obesos (Quadro 4). Em um artigo a presença de distúrbio alimentar decorrente de um mecanismo compensatório dos sintomas depressivos também esteve correlacionado ao desenvolvimento da obesidade e baixa autoestima. A autoimagem dos adolescentes tem impacto fundamental em sua autoestima e, consequentemente, no desenvolvimento de transtornos de humor.

\section{Discussão}

Durante a análise dos artigos selecionados observou-se que os aspectos abordados contribuíam direta ou indiretamente com a relação entre sintomas depressivos e a obesidade. Dentre esses aspectos foram observados três fatores recorrentes para o estabelecimento deste vínculo entre ambas patologias, foram eles: a percepção da imagem corporal, o ambiente social no qual o jovem está incluído e os hábitos de cuidado pessoal que esse adolescente tem consigo. 
Research, Society and Development, v. 10, n. 9, e48910918185, 2021

(CC BY 4.0) | ISSN 2525-3409 | DOI: http://dx.doi.org/10.33448/rsd-v10i9.18185

Em uma meta-análise realizada na China descobriu-se que sintomas depressivos são comuns em crianças e adolescentes com sobrepeso e obesos em sua população 24,02\% (IC 95\%: 15,92\% - 33,16\%, I2 = 97,9\%), com esses indivíduos apresentando uma chance de quase duas vezes mais de apresentar sintomas depressivos quando obesos depressivos $(\mathrm{OR}=1,877$, IC 95\%: 1,459-2,415, I2 =77,1\%, P <0,001), o que estava de acordo com estudos ocidentais e mundiais sobre o tema (Rao et al., 2019).

Estatisticamente, durante a observação da população em análise nesses estudos foi evidenciado que adolescentes, apresentando ou não obesidade, manifestaram ao menos um transtorno de humor durante esse período da vida (Aguilar, 2018). Em um estudo foi encontrado mais sintomas depressivos em meninas que em meninos (7,0\% vs. 4,8\%; p <0,0001), mas o surgimento desses sintomas aparecia mais precocemente em meninos, cerca de 8 meses em relação à idade (Lindberg, 2020). Outro estudo realizado com adolescentes negros também encontrou prevalência de sintomas depressivos mais elevadas em mulheres (média de 6,00 vs. 2,31, p = 0,080), porém não houve diferenças na insatisfação corporal por sexo (Wang, 2020).

Porém em um estudo chinês apenas adolescentes do sexo masculino com depressão, mas não do sexo feminino, tiveram um risco maior de sobrepeso ou obesidade, independentemente de a depressão ter sido medida com uma variável binária (CES$\mathrm{D} \geq 16$ ) ou contínua (escores CES-D), concluindo que a depressão estava significativamente associada à obesidade entre homens e não entre mulheres (Zhao, 2019)

A percepção da imagem corporal foi o fator mais associado como preditor entre a depressão e a obesidade. Os artigos 5 e 7, mencionados no quadro de classificação, introduzem esse tema ao referirem que 1 a cada 9 adolescentes apresentam insatisfação corporal (Aguilar, 2018) e isso muito se deve a autopercepção física que esses adolescentes têm do corpo (Ocampo Bustos, 2017).

A percepção errônea que os jovens apresentam sobre seu corpo podem levar a duas vertentes. A primeira delas diz respeito aos adolescentes que tem obesidade e ao se avaliarem de maneira equivocada, ou seja, não perceberem seu real peso apresentavam menos sintomas depressivos do que os adolescentes que tinham uma autopercepção geral do seu corpo tal como era (Thurston, 2017). Por outro lado, a noção prejudicada da imagem corporal leva a variações da autoestima do adolescente (Rentz-Fernandes, 2017) e os efeitos negativos dessa baixa autoestima reflete em uma maior tendência de sintomas depressivos.

Um estudo encontrou evidências de que adolescentes negros são menos vulneráveis às pressões relacionadas ao ideal de magreza, em comparação com adolescentes brancos. Além disso, mais de três quartos das mães dos adolescentes negros de baixa renda avaliados nesse estudo estavam ou com sobrepeso ou obesas, podendo indicar que adolescentes negros de baixa renda então protegidos do ideal de magreza (Wang, 2020).

Em comum acordo com essas variações de autoestima, Esmaeilzadeh (2018) apresentou dados que comprovam que os maus hábitos, como a ausência de atividades físicas regulares e nutrição inadequada, adotados por esses adolescentes contribuem para o aparecimento de tais sintomas. Aliada a esses dois fatores está o estigma social no qual o adolescente é imposto associado ao aumento da exposição as mídias sociais, tais pontos cooperam para que o padrão de "mulheres magras e homens musculosos" sejam perpetuados por esse meio, satisfazendo positivamente a taxa crescente de adolescentes com depressão (Quek, 2017).

Por muitas vezes, esses jovens não apresentam o acompanhamento multidisciplinar adequado, para entender quais fatores estão predispondo os transtornos de humor e a obesidade. Desta forma, acabam frustrados e estressados tendo como válvula de escape, muitas vezes, para os sintomas depressivos os transtornos alimentares compulsivos (Goldschmidt, 2015). A compulsão alimentar, por sua vez, colabora para o excesso de peso que gradualmente desaponta em uma obesidade, o adolescente, então, devido aos efeitos negativos que a patologia pode acarretar - baixa autoestima - apresenta os persistentes sintomas depressivos. O adolescente, por fim, entra em um ciclo vicioso tendo como principais pilares o estigma social, a autopercepção corporal e os maus hábitos, fortalecendo a relação entre sintomas depressivos e a obesidade. 
Research, Society and Development, v. 10, n. 9, e48910918185, 2021

(CC BY 4.0) | ISSN 2525-3409 | DOI: http://dx.doi.org/10.33448/rsd-v10i9.18185

\section{Conclusão}

O presente estudo auxilia na compreensão dos principais fatores apontados na literatura como percursores da relação entre sintomas depressivos e obesidade. Ademais, serão necessários estudos futuros para um melhor entendimento das outras possíveis variáveis que podem influenciar nesse vínculo, bem como um maior aprofundamento das associações encontradas e da abordagem da equipe multidisciplinar para uma melhor interação com os adolescentes a fim de melhorar seu cuidado.

\section{Referências}

Aguilar Aguilar, C. K., Blanco Castillo, L., Villarreal Ríos, E., Vargas Daza, E. R., Galicia Rodríguez, L., \& Martínez González, L. (2018). Asociación de sobrepeso u obesidad con trastornos del estado de ánimo en adolescentes. Archivos latinoamericanos de nutrición, $321-327$.

Esmaeilzadeh, S., Farzizadeh, R., Kalantari, H. A., Mahmoudi, A., Bilehsavar, O. Y., \& Mehranpour, A. (2018). Central or overall obesity: which one is a better predictor of depressive symptoms in children, adolescents, and youths?. Eating and Weight Disorders-Studies on Anorexia, Bulimia and Obesity, 23(1), 117123.

Goldschmidt, A. B., Wall, M. M., Choo, T. J., Larson, N. I., \& Neumark-Sztainer, D. (2015). Mediators involved in the relation between depressive symptoms and weight status in female adolescents and young adults. International Journal of Obesity, 39(6), 1027-1029.

Gutiérrez-Sánchez, G., Salazar-Barajas, M. E., Ruiz-Cerino, J. M., Ávila-Alpirez, H., de la Luz Martínez-Aguilar, M., \& Guerra-Ordoñez, J. A. (2019). Depresión como factor asociado a la obesidad en adolescentes/Depression as a factor associated to obesity in adolescents/Depressão como fator associado à obesidade em adolescentes. Journal Health Npeps, 4(2), 16-27.

Lindberg, L., Hagman, E., Danielsson, P., Marcus, C., \& Persson, M. (2020). Anxiety and depression in children and adolescents with obesity: a nationwide study in Sweden. BMC medicine, 18(1), 1-9.

Ocampo, J., Guerrero, M., Espín, L., Guerrero, C., \& Aguirre, R. (2017). Asociación entre índice de masa corporal y depresión en mujeres adolescentes. International Journal of Morphology, 35(4), 1547-1552

ONU. Agências da ONU discutem como reduzir transtornos de saúde mental de crianças e adolescentes. 2019. https://news.un.org/pt/story/2019/11/1693471.

Organização Mundial de Saúde (OMS). Aumenta o número de pessoas com depressão no mundo. 2017. https://www.paho.org/bra/index.php?option=com_content\&view=article\&id=5354:aumenta-o-numero-de-pessoas-com-depressao-no-mundo\&Itemid=839.

Organização Mundial de Saúde (OMS). o Brasil, países das Américas definem próximos passos para o enfrentamento da epidemia de obesidade infantil. 2019. https://www.paho.org/bra/index.php?option=com_content\&view=article\&id=5957:no-brasil-paises-das-americas-definem-proximos-passos-para-oenfrentamento-da-epidemia-de-obesidade-infantil\&Itemid=839

Organização Mundial de Saúde (OMS). Obesidade entre crianças e adolescentes aumentou dez vezes em quatro décadas, revela novo estudo do Imperial College London e da OMS. 2017. https://www.paho.org/bra/index.php?option=com_content\&view=article\&id=5527:obesidade-entre-criancas-e-adolescentesaumentou-dez-vezes-em-quatro-decadas-revela-novo-estudo-do-imperial-college-london-e-da-oms \&Itemid=820.

Quek, Y. H., Tam, W. W., Zhang, M. W., \& Ho, R. C. (2017). Exploring the association between childhood and adolescent obesity and depression: a metaanalysis. Obesity reviews, $18(7), 742-754$.

Rao, W. W., Zhang, J. W., Zong, Q. Q., An, F. R., Ungvari, G. S., Balbuena, L., ... \& Xiang, Y. T. (2019). Prevalence of depressive symptoms in overweight and obese children and adolescents in mainland China: A meta-analysis of comparative studies and epidemiological surveys. Journal of affective disorders, 250, 26-34.

Rentz-Fernandes, A. R., Silveira-Viana, M. D., Liz, C. M. D., \& Andrade, A. (2017). Autoestima, imagem corporal e depressão de adolescentes em diferentes estados nutricionais. Revista de salud pública, 19, 66-72.

Roberts, R. E., \& Duong, H. T. (2015). Does major depression affect risk for adolescent obesity?. Journal of affective disorders, $186,162-167$.

Sadock, B. J., Sadock, V. A., \& Ruiz, P. (2016). Compêndio de Psiquiatria-: Ciência do Comportamento e Psiquiatria Clínica. Artmed Editora.

Salles, L. M. F. (2005). Infância e adolescência na sociedade contemporânea: alguns apontamentos. Estudos de Psicologia (Campinas), 22(1), 33-41.

Soares, C. B., Hoga, L. A., Peduzzi, M., Sangaleti, C., Yonekura, T., Silva, D. R. A. D., \& Trevizan, M. A. (2010). Revisão Integrativa versus Revisão Sistemática. Reme: Revista Mineira de Enfermagem, 8(1), 102-106.

Thurston, I. B., Sonneville, K. R., Milliren, C. E., Kamody, R. C., Gooding, H. C., \& Richmond, T. K. (2017). Cross-sectional and prospective examination of weight misperception and depressive symptoms among youth with overweight and obesity. Prevention Science, $18(2), 152-163$.

Wang, Y., Lynne, S. D., Witherspoon, D., \& Black, M. M. (2020). Longitudinal bidirectional relations between body dissatisfaction and depressive symptoms among Black adolescents: A cross-lagged panel analysis. PloS one, 15(1), e0228585.

Zhao, Z., Ding, N., Song, S., Liu, Y., \& Wen, D. (2019). Association between depression and overweight in Chinese adolescents: a cross-sectional study. BMJ open, $9(2), \mathrm{e} 024177$. 